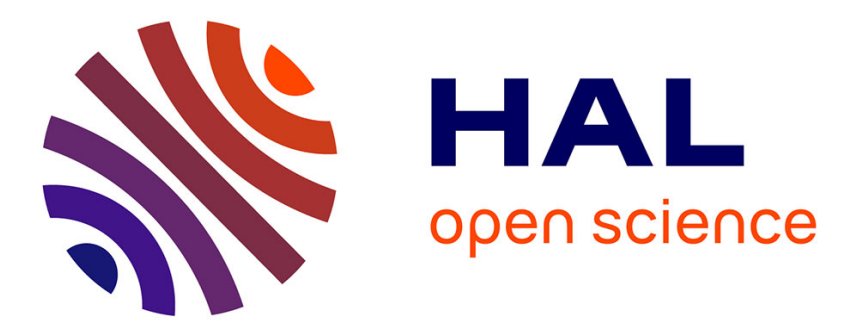

\title{
X-ray diffraction and stress relaxations to study thermal and stress-assisted annealings in nanocrystalline gold thin films
}

\author{
P. Godard, D. Faurie, T. Sadat, M. Drouet, D. Thiaudiere, P.O. Renault
}

\section{- To cite this version:}

P. Godard, D. Faurie, T. Sadat, M. Drouet, D. Thiaudiere, et al.. X-ray diffraction and stress relaxations to study thermal and stress-assisted annealings in nanocrystalline gold thin films. Acta Materialia, 2019, 173, pp.87-95. 10.1016/j.actamat.2019.04.024 . hal-02285914

\author{
HAL Id: hal-02285914 \\ https://hal.science/hal-02285914
}

Submitted on 22 Oct 2021

HAL is a multi-disciplinary open access archive for the deposit and dissemination of scientific research documents, whether they are published or not. The documents may come from teaching and research institutions in France or abroad, or from public or private research centers.
L'archive ouverte pluridisciplinaire HAL, est destinée au dépôt et à la diffusion de documents scientifiques de niveau recherche, publiés ou non, émanant des établissements d'enseignement et de recherche français ou étrangers, des laboratoires publics ou privés.

\section{(ㄷ)(1) $\$$}

Distributed under a Creative Commons Attribution - NonCommerciall 4.0 International 


\title{
X-Ray Diffraction and Stress Relaxations to Study Thermal and Stress-assisted Annealings in Nanocrystalline Gold Thin Films
}

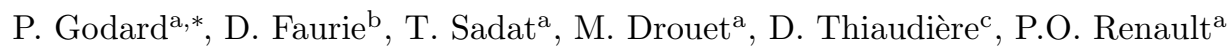 \\ ${ }^{a}$ CNRS - Université de Poitiers ENSMA, Futuroscope Chasseneuil, France \\ ${ }^{b}$ LSPM - CNRS, Université Paris13, Université Sorbonne Paris Cité, Villetaneuse, France \\ 'Synchrotron SOLEIL, L'Orme des Merisiers, Saint-Aubin, Gif-sur-Yvette Cedex, France
}

\begin{abstract}
The dependence on thermal history of the plasticity mechanisms occurring in nanocrystalline gold thin films is evidenced thanks to relaxation tests combined with in-situ synchrotron X-ray diffraction. The two techniques complement one another. The activation parameters show that the films deform mainly by dislocations and give indications about their mean free paths, whereas the Bragg peak positions, widths and areas bring invaluable information on residual stress as well as on some plasticity properties, like dislocation storage inside the grains or grain rotations. For the demonstration, two sputter-deposited nanocrystalline $50 \mathrm{~nm}$-thin films deposited onto stretchable substrates are studied. It is shown that an as-grown sample (at a homologous temperature of 0.22 ) presents a stress-assisted annealing, thus decreasing its initial defect density, whereas if a thermal annealing (three hours at $200^{\circ} \mathrm{C}$, corresponding to a homologous temperature of 0.35 ) has been applied to the sample before the tensile test, it deforms by conventional plasticity, and the dislocations are not stored inside the grains. These mechanisms lead to different work-hardening properties. This work shows how a moderate annealing can have a profound influence on the mechanical behaviour of these thin films.
\end{abstract}

Keywords: Relaxation, Nanocrystalline film, Annealing, Microstructure, X-ray diffraction

\section{Introduction}

The plastic behaviour of a material subjected to a mechanical test results from the synergy between applied stress and thermal energy. To separate both effects, transient tests are resorted to: after an initial mechanical perturbation, the stress or the strain is kept constant and the material is let evolve towards a new equilibrium configuration, with only thermal fluctuations assisting plastic events. It is then convenient to separate the stress exerted on the material in an athermal contribution $\tau_{\mu}$ and a thermal one $\tau^{*}$. A. Seeger in 1954 identified the first with the internal stress that is governed by long range stresses associated to grain boundaries, precipitates, dislocation walls, etc., and the second with the effective stress caused by much more localized heterogeneities, like point defects or isolated dislocations. The dependence on the effective stress of the activation energy of a plastic event is monitored with the effective activation volume $V^{*}$ :

$$
V^{*}=-\left.\frac{\partial \Delta G}{\partial \tau^{*}}\right|_{T}
$$

This volume is associated to the number of atoms participating to the plastic events and is thus characteristic of

*Corresponding author. SP2MI - Téléport 2, 11 Boulevard Marie et Pierre Curie, BP 30179, F86962 Futuroscope Chasseneuil Cedex, France, Email address: pierre.godard@univ-poitiers.fr the leading plasticity mechanism $[1,2,3,4]$. There exists another activation volume, $V_{a}$, termed the apparent activation volume. It is measured in practice with the decay of the stress during the relaxation time as $[5,6]$

$$
\Delta \tau(t)=-\frac{k T}{V_{a}} \ln (1+t / c)
$$

with $t$ the time, $k$ the Boltzmann constant and $T$ the absolute temperature - or by the strain decay during the creep time, i.e. when the applied stress rate vanishes. The apparent activation volume differs from the effective (also called physical, or true) activation volume in that the microstructure may slightly evolve during the relaxation segment. Hence, $V_{a}$ not only depends on thermal activation, but also on the small change in internal stress. Though experimental means exist to extract the effective activation volume $V^{*}$ from the apparent activation volume $V_{a}$, e.g. through repeated relaxation tests done at the same stress $[7,8]$, numerous authors use $V_{a}$ as a first guide for identification of the plasticity mechanisms [9, 10, 11, 12, 13].

Relaxation tests are particularly well adapted for nanocrystalline materials, for which several mechanisms are in competition $[14,15]$ : many studies reveal dislocation nucleations and propagations [16, 17, 18], diffusion $[19,20,21]$, grain boundary sliding, migration, rotation or growth $[22,23,24,25,26,27]$ - these mechanisms often cooperate and accommodate one another. It is hoped that the activation volume determination will be a method to 

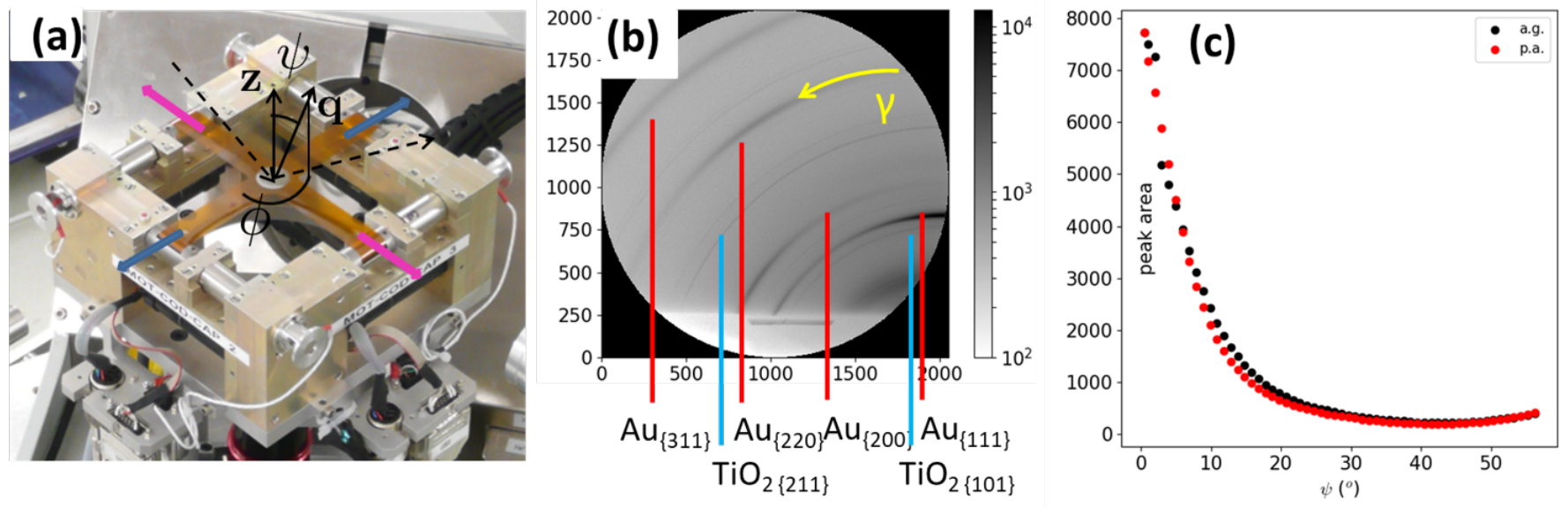

Figure 1: (a): Picture of the biaxial tensile tester available at the DiffAbs beamline. The substrate arm width and length is 20 and $90 \mathrm{~mm}$ respectively, and the diameter of the disk-shaped thin film is $20 \mathrm{~mm}$. (b): Two-dimensional diffractogram, with portions of the Debye-Scherrer rings of the gold $(\mathrm{Au})$ thin film and the powder used as a calibrant $\left(\mathrm{TiO}_{2}\right)$. Only the indexed peaks are used for the calibration. (c): Peak area of the $\{111\}$ pole for the two samples. In all the figures of this paper, black applies to the as-grown (a.g.) sample, red to the post-annealed (p.a.) one.

characterize macroscopically the leading plasticity mechanism operating in the material $[28,29,10,30,31,32,33]$. Moreover, the thermodynamic instability of nanocrystalline metals being evidenced by the potential grain growth $[34,23]$, it is relevant to monitor the relaxation of a nanocrystalline sample all along a stress-strain curve. In this paper we are interested in thermal contributions in the mechanical behaviours of nanocrystalline gold thin films deposited onto soft substrates (polyimides). First, one of the films has been annealed after its deposition. This did not change its grain size but affected its defect content. Second, relaxations have been imposed on the films and their responses compared. For these samples, the macroscopic data do not reflect the stress in the film, and synchrotron X-Ray Diffraction (XRD) is resorted to. This provides the elastic strain, of which the residual and applied stresses can be determined. Simultaneously, the true strain has been monitored with Digital Image Correlation (DIC). As shown in [35], the evolution of the Bragg peak shape during the loading-relaxation test brings complementary information on the mechanical response of the samples. The relevance of XRD data analysis for characterizing the plasticity behaviours of nanocrystalline materials was already shown in e.g. $[36,37]$ (Bragg peak width evolution), [38] (asymmetry), or [39, 40] (texture).

Very recently, the relaxation of nanocrystalline and ultrafine grained gold thin films has been the subject of several studies: Hosseinian et al observed with in-situ transmission electron microscopy that the relaxation was first accommodated by dislocation motion, mainly around triple junctions involving relatively large grains, and then with grain boundary diffusion mechanisms [13], whereas Mongkolsuttirat et al show that the relaxation was more substantial when the temperature or the grain size increase [41]. We show here that the initial microstructure (defect content and residual stress) has also to be taken into account. Besides, there has been recently a marked interest in characterizing through diffraction biaxial tests $[42,43,44]$. Biaxial mechanical testing configurations can indeed provide much more information about the anisotropic stress-strain behaviour than uniaxial studies. But, in the present manuscript, the biaxial test is not mandatory because the thin films are transversally isotropic (due to the fibre texture). The mechanical properties are thus isotropic in the plane stress state.

\section{Experimental}

\subsection{Sample preparation}

Two gold thin films have been simultaneously prepared by physical vapor deposition on a $125 \mu \mathrm{m}$-thick polyimide substrate (Kapton ${ }^{\circledR}$ ) at room temperature. The pressure before deposition was $510^{-7}$ mbar and increases to $1.410^{-4}$ mbar during deposition. The 13 minutes deposition resulted in $49.5 \pm 0.5 \mathrm{~nm}$-thick gold films, as measured with visible light profilometry and X-ray reflectometry. One of the films has been annealed for 3 hours at $200^{\circ} \mathrm{C}$. Both films have columnar grains, with an average grain diameter of $50-70 \mathrm{~nm}$. Grains are thus almost equiaxed. The films are $\{111\}$ fibre-textured, as shown in the two-dimensional X-ray diffractogram of Fig. $1 \mathrm{~b}$ and on the diffracting volumes integrated along the $\{111\}$ ring in Fig. 1c. At small scale, i.e. within a field of view of $500 \times 500 \mathrm{~nm}^{2}$, the rms surface roughness was $1.46 \mathrm{~nm}$, as measured with atomic force microscopy.

\subsection{Strain measurements}

A picture of the specimen (cruciform stretchable substrate and gold thin film) mounted on the biaxial tensile tester is presented in Fig. 1a. This tensile tester is available at the DiffAbs beamline of the synchrotron radiation 

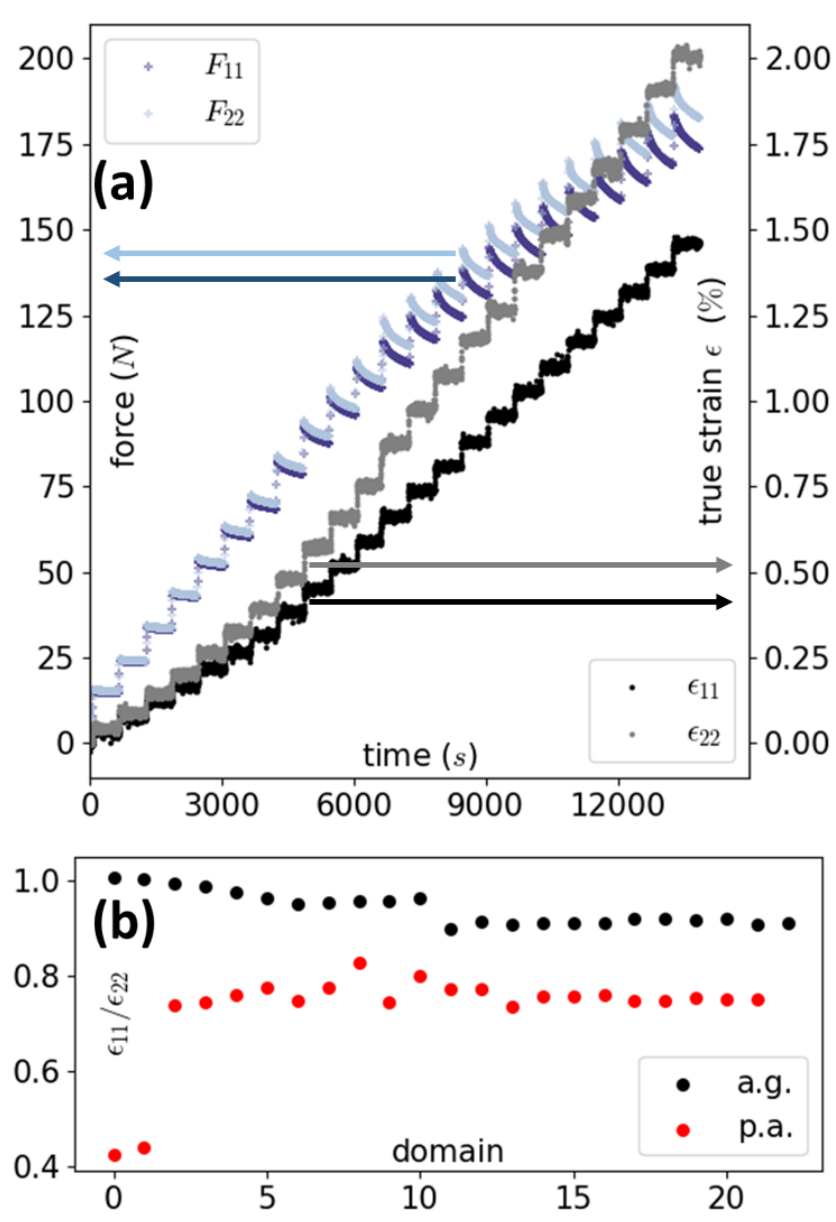

Figure 2: (a): Forces applied on the cruciform substrate and the resulting true strain at the centre of the cruciform shape composite. After a displacement jump, the force decreases whereas the true strain remains constant. (b): Ratio between the two principal strain components.

facility SOLEIL [45]. It comprises four independent motors and four load sensors, that allow to perform tensile tests with different (and not necessarily constant) load ratios. Below the machine lies a microscope objective and a charge-coupled device camera for the true strain measurement with DIC analysis ( $q 4$-Correli software [46]). The grey levels are obtained by applying paint speckles on the rear surface of the substrate, and this paint contains crystallites of $\mathrm{TiO}_{2}$ in the rutile phase. With X-rays this gives continuous Debye-Scherrer rings that allow a calibration of the diffraction parameters during the test.

The in-situ XRD measurements were performed with a beam energy of $16.00 \mathrm{keV}$. Images were recorded every 5 seconds with a two-dimensional detector, Fig. 1b, a Mar 165-SX lying $264 \mathrm{~mm}$ from the sample. The beam width is approximately $250 \times 300 \mu^{2}$, vertical times horizontal, but the incident angle being $9^{\circ}$, the footprint spreads to $1600 \times 300 \mu \mathrm{m}^{2}$. Note that this footprint remains within the homogeneous strained area at the centre of the cruciform substrate [45]. For circular grains with an average grain diameter of $50 \mathrm{~nm}$, about $2.510^{8}$ grains are simultaneously illuminated. This insures a high representativity of these X-ray results.

\subsection{Tensile relaxation test}

Fig. 2a presents the tensile test of the as-grown sample from a macroscopic point of view. The test of the postannealed sample was very similar. These are displacementcontrolled tests: every ten minutes, an elongation is imposed to the Kapton. This leads to a load and a strain jumps. As already reported in [47] and [35], after a jump, the force exerted by the composite on the tester decreases whereas the true strain at the centre of the cruciform shape composite remains constant, with a standard deviation smaller than $710^{-5}$ (note that this value includes the noise induced by the DIC algorithm). In other words, the substrate imposes a constant applied strain to the thin film as shown in fig. 2a (cf strain plateaus). As also established in our previous works, the strain is fully transmitted through the film-substrate interface [47, 48]. This allows measuring by X-ray diffraction what happens versus time in the polycrystalline gold film deposited at the centre of the cruciform shape substrate while the macroscopic applied strain is constant: it is therefore a relaxation test.

The displacements imposed to the Kapton are the same on the two axes. Nevertheless, because of the mechanical anisotropy of the Kapton, the ratio of true strains at the centre of the cruciform substrate lies between 0.91 and 1.0 for the as-grown sample, 0.74 and 0.83 for the postannealed one (except, for the latter, at the very beginning of the test). Following a von Mises yield surface, the small difference of loading paths (ratio of $\epsilon_{11} / \epsilon_{22}=0.95 \pm 0.05$ versus $0.79 \pm 0.05$ ) allows assuming a yield point that is directly comparable. The deformation tests may be considered as similar. Before the deformation test per se, an equibiaxial initial load of $15 \mathrm{~N}$ was applied. This was probably insufficient for the post-annealed sample, since the first two relaxation segments have a strain ratio smaller than 0.5 , see Fig. 2b. As usual, we will term relaxation segment a plateau of constant true strain, and assign to it the mean with respect to time of the average of the two principal true strain components. The relaxation segments lasted for slightly more than 500 seconds, and the strain rate from one relaxation segment to the subsequent one was $3.4 \pm 1.210^{-5} s^{-1}$. A test up to $178 N($ resp. $180 N)$ was uniformly split into 23 (resp. 22) relaxation segments for the as-grown (resp. post-annealed) sample.

\section{Data analysis and Results}

\subsection{Stress and activation volumes measurements}

The direction of a scattering vector $\mathbf{q}$ is described with $\psi$, the angle between $\mathbf{q}$ and the specimen surface normal, and $\phi$ its azimuthal coordinate. The use of a twodimensional detector allows to record simultaneously portions of Debye-Scherrer rings for several lattice plane families. Let $\gamma$ be the angle along the Debye-Scherrer ring of 

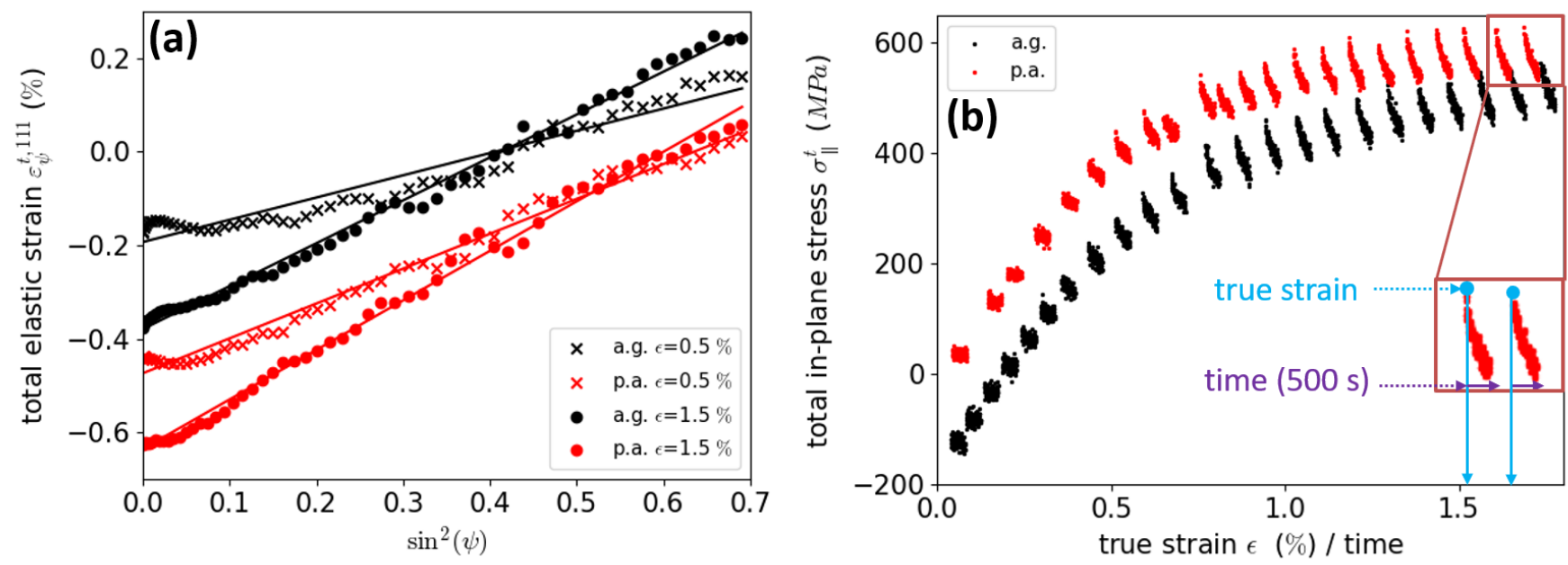

Figure 3: (a): $\varepsilon^{t, 111}-\sin ^{2} \psi$ curves for the first point of the relaxation segment with true strains closest to $\epsilon=0.5 \%$ and $\epsilon=1.5 \%$. (b): Total stress deduced from (a) as a function of true strain and time. Because the true strain is constant on a relaxation segment the first point of each relaxation segment has the abscissa of the true strain, the other points inside the relaxation segment have abscissas that correspond to time, with $5 s$ corresponding to $410^{-4} \%$ (see two relaxation segments in the inset). We observe that the annealing reduced the residual stress to zero.

one lattice plane family, as shown in Fig. 1b. As detailed in [49], $\psi$ and $\phi$ depend on $\gamma$, the Bragg angle $\theta^{h k l}$ and the incidence angle $\omega$. Thanks to the Bragg's law, one is able to compute the total (applied plus residual) elastic strain

$$
\varepsilon_{\psi, \phi}^{t, h k l}=\frac{d_{\psi, \phi}^{h k l}-d_{0}^{h k l}}{d_{0}^{h k l}}
$$

with $d_{0}^{h k l}$ the bulk lattice parameter, $d_{0}^{100}=0.40782 \mathrm{~nm}$ for gold. However, because the test is equibiaxial, and because a huge number of grains are simultaneously probed for each scattering direction, a probed strain component is in fact a mean over the in-plane orientations, so the subscript $\phi$ is no more specified. Some $\varepsilon^{t, 111}-\sin ^{2} \psi$ curves are exhibited in Fig. 3a. For each sample, two curves are highlighted, those corresponding to the first point of the relaxation segment with true strains as close as possible to $\epsilon=0.5 \%$ and to $\epsilon=1.5 \%$. One notes that for a given true strain, the out-of-plane elastic strain (at $\psi=0^{\circ}$ ) of the post-annealed sample is more compressive than for the asgrown sample, and the total in-plane stress (proportional to the slope of the curve) is more tensile. Also, a slight non-linearity is observed, probably due to anisotropic elastic [50] and plastic grain interactions in such thin films with columnar grains. From the slope $s^{h k l}$ of the linear fit of the $\varepsilon^{t, h k l}-\sin ^{2} \psi$ curve, one deduces the total in-plane stress $\sigma_{\|}^{t}:=\sigma_{\psi=90^{\circ}}^{t}$ from

$$
\sigma_{\|}^{t}=\frac{E^{h k l} s^{h k l}}{1+\nu^{h k l}}
$$

where $E^{h k l}$ and $\nu^{h k l}$ are the orientation dependent Young modulus and Poisson ratio. The evolution of the stress in the two samples is presented in Fig. 3b, with elastic constants corresponding to fibre-textured gold thin films:
$E^{111}=90 G P a, \nu^{111}=0,53[51]$. It is seen that the residual stress of the as-grown sample is approximately $-200 M P a$, and that the annealing reduces this value to about zero.

The experimental data points showing the stress evolution shown in Fig. 3b are too scattered for the fit to determine the activation volume within a sufficiently precise value. This scatter is probably induced both by the nonlinearity of the $\varepsilon^{t}-\sin ^{2} \psi$ plots and the crystallographic texture of the gold film. Indeed, the intensity of the Bragg peak is much smaller at $\psi=45^{\circ}$ than $\psi=0^{\circ}$ (from the integrated Bragg peak of Fig. 1c there are about 40 more numerous grains scattering at $\psi=0^{\circ}$ than at $\psi=45^{\circ}$ ). If only Bragg peaks with a high signal-to-noise ratio are used, the absolute stress value may contain a significant error of about $20 \%$, but the relative stress change can be measured very precisely. So, we propose to use the intercept of the $\varepsilon^{t, 111}-\sin ^{2} \psi$ curves, with the hypotheses that the out-of-plane stress vanishes in these ultra-thin films and that the tests are equibiaxial. Denoting by $\varepsilon_{\psi}^{h k l}$ without the subscript $t$ the applied strain, that is the strain obtained with Eq. 3 when $d_{0}^{h k l}$ is replaced by $d_{\psi}^{h k l}$ taken at the beginning of the test, Fig. 4a presents $\varepsilon_{\perp}^{111}:=\varepsilon_{\psi=0}^{111}$ 。 as a function of true strain and time. Examples are shown in Fig. 4b, for a true strain of $\epsilon=0.3 \%, 0.5 \%$ and $1.5 \%$. The fit of the out-of-plane strain is now very good, at least once $\epsilon \gtrsim 0.25 \%$; A logarithmic decay is used:

$$
\Delta \varepsilon_{\perp}^{h k l}(t)=\frac{k T}{D^{h k l}} \ln \left(1+t / c^{h k l}\right)
$$

with two fitting parameters, the prefactor $D^{h k l}$ and the time constant $c^{h k l}$ - both depend on the elastic constants and so on the probed reflection. In appendix, we demonstrate, with all the underlined hypotheses, that the loga- 

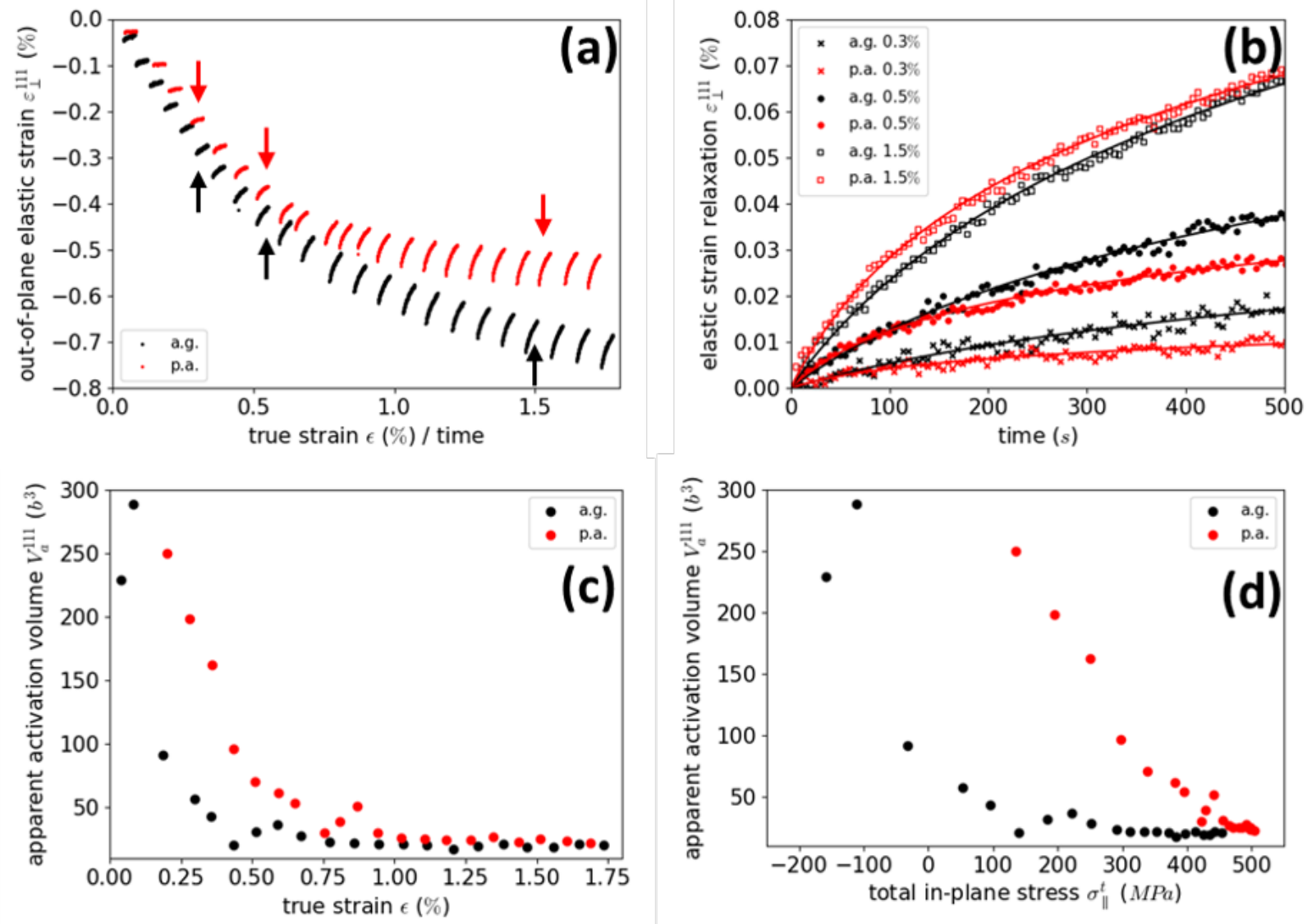

Figure 4: (a): Applied out-of-plane elastic strain $\varepsilon_{\perp}^{111}$ as a function of true strain and time (same abscissa as Fig. 3b). The arrows point to the curves exemplified in (b). (b): Relaxation of $\varepsilon_{\perp}^{\frac{1}{11}}$ as a function of time and fit with a logarithmic decay. The match is very satisfying. (c): Apparent activation volume $V_{a}^{111}$ as a function of true strain $\epsilon$. (d): Apparent activation volume $V_{a}^{111}$ as a function of the total stress at the beginning of the relaxation state $\sigma_{\|}^{t}(t=0)$. The effect of the annealing is more clearly exhibited in this subfigure.

rithmic decay applies in the case under study. The maximum deviation between the measurement and the fit of $\varepsilon_{\perp}^{111}$ is $310^{-5}$. Now the in-plane strain is deduced and the in-plane stress with Hooke's law:

$$
\sigma_{\|}=\frac{-E^{h k l}}{2 \nu^{h k l}} \varepsilon_{\perp}^{h k l}
$$

Converting the normal stress $\sigma_{\|}$to a shear stress $\tau$ with a Taylor factor $M$, one arrives at the following expression for the apparent activation volume:

$$
V_{a}=\frac{2 M \nu^{h k l} D^{h k l}}{E^{h k l}} .
$$

At room temperature, and with a Taylor factor midway between $\sqrt{3}[10,52,53,18]$ and 3 (close to the value corresponding to coarse-grained polycrystals, but also used for nanocrystals [28, 39]), one obtains the curves of Fig. 4c. Even if the absolute error may be as large as $25 \%$, the behaviour is clear: for both samples the apparent activation volumes first decrease and stagnate, from $\epsilon \approx 0.7 \%$ for the as-grown sample and from $\epsilon \approx 1.0 \%$ for the post-annealed one. Moreover, at the beginning of the test, the apparent activation volumes are much larger for the latter than for the former.

The apparent activation volumes are presented as functions of the total in-plane stress in Fig. 4d. The importance of taking into consideration the residual stress is evident from this figure.

\subsection{Bragg peak shape evolution}

In-situ XRD not only provides the evolution of stress in a material, but also microstrain and texture evolutions. The Bragg peaks are correctly fitted with a linear background and the following asymmetric Pearson VII function:

$$
\begin{aligned}
& P_{I_{0}, 2 \theta_{B}, w, m, A}(2 \theta)= \\
& =H_{2 \theta_{B}}(2 \theta) I_{0}\left[1+\left(\frac{2\left(2 \theta-2 \theta_{B}\right)}{1-A}\right)^{2} \frac{\left(2^{1 / m}-1\right)}{w^{2}}\right]^{-m} \\
& +H_{-2 \theta_{B}}(-2 \theta) I_{0}\left[1+\left(\frac{2\left(2 \theta-2 \theta_{B}\right)}{1+A}\right)^{2} \frac{\left(2^{1 / m}-1\right)}{w^{2}}\right]^{-m}
\end{aligned}
$$



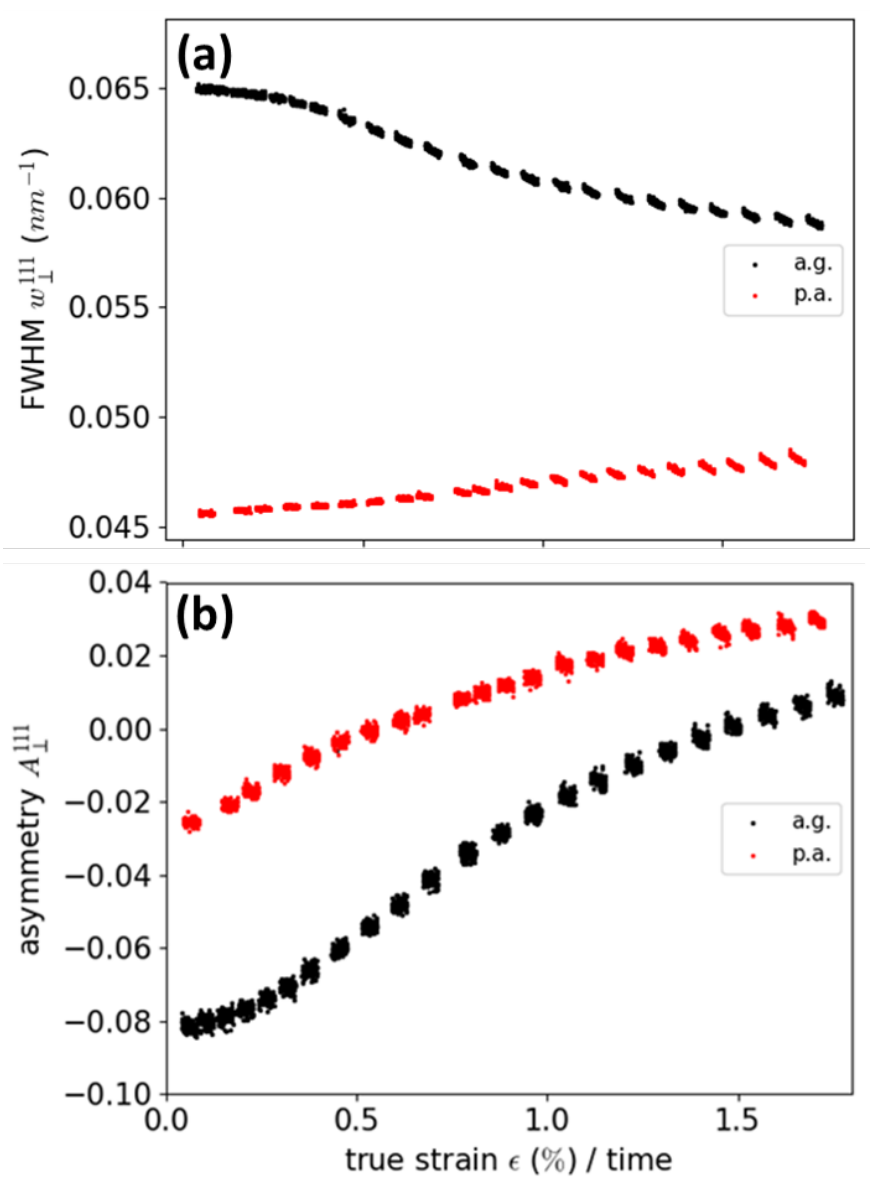

Figure 5: Evolution of the parameters of the peak fit for the pole (i.e. at $\psi=0^{\circ}$ ) of the $\{111\}$ diffracting plane family (a): Full width at half-maximum. (b): Asymmetry parameter. Same abscissa as Fig. 3b.

with $H_{x}$ the Heaviside function with a step at $x, 2 \theta_{B}$ the Bragg angle, $I_{0}$ the maximum of the peak intensity, $w$ the full width at half maximum, $A$ the asymmetry parameter (which vanishes for a symmetric function) and a parameter $m$ that controls the kurtosis, i.e. the tail of the peak in our case. Let $\theta_{l}$ and $\theta_{h}$ be such that $P\left(2 \theta_{l}\right)=P\left(2 \theta_{h}\right)=I_{0} / 2$ and $2 \theta_{l}<2 \theta_{B}<2 \theta_{h}$ - subscripts stand for low and high parts of the peak. The half widths at half maximum are $v_{l}=2 \theta_{B}-2 \theta_{l}=\frac{w}{2}(1+A)$ and $v_{h}=2 \theta_{h}-2 \theta_{B}=\frac{w}{2}(1-A)$ respectively, so that $A=\frac{v_{l}-v_{h}}{v_{l}+v_{h}}$. For further use, the area under the fitted Bragg peak is [54]

$$
\int_{\mathbb{R}} P_{I_{0}, 2 \theta_{B}, w, m, A}(2 \theta) d(2 \theta)=\frac{I_{0} w \pi^{1 / 2} \Gamma(m-1 / 2)}{2 \Gamma(m)\left(2^{1 / m}-1\right)^{1 / 2}}
$$

where $\Gamma$ is the gamma function. We note that the peak area does not depend on $A$, and that it is much less noisy to plot the area of the fitted peak than the area of the measured peak.

The parameters $w_{\perp}^{111}$ and $A_{\perp}^{111}$ are shown in Fig. 5 . To ease comparison with other studies, the FWHM is expressed in $\mathrm{nm}^{-1}$ from the scattering vector expression $q=2 \sin (\theta / \lambda)$. The first striking remark is the initial value of the full width at half maximum: the thermal annealing lowered $w_{\perp}^{111}$ by $30 \%$, from $0.292^{\circ}$ to $0.205^{\circ}$. Correspondingly, the intensity $I_{0, \perp}^{111}$ is almost twice as large for the post-annealed than for the as-grown sample (not shown). Quite interestingly, one also notes that whereas $w_{\perp}^{111}$ decreases along the test for the as-grown sample, it increases for the post-annealed one. Nevertheless, inside the relaxation segments, it slightly decreases for the two samples. This is because plastic events lower intergrain (type II) and/or intragrain (type III) elastic strain heterogeneities. The maximum of the intensity, $I_{0, \perp}^{111}$ remains constant (resp. increases) for the post-annealed (resp. asgrown) sample, but tends to increase inside relaxation segments for both samples. Finally, for both samples, the asymmetry increases, even if the values remain small.

\section{Discussion}

Based on the experimental data from in-situ synchrotron X-ray diffraction experiments, the probable plasticity mechanisms occurring in these two films are now discussed. Let us remind that X-ray diffraction analysis, though leading possibly to significant error bars of about $20 \%$ in the absolute stress values when the stress is deduced from $\varepsilon_{\perp}^{111}$ alone, captures quite nicely the relative stress changes. Hence, X-ray diffraction brings valuable complementary information as opposed to conventional relaxation tests, namely mechanical macroscopic measurements or inside an electron microscope. Moreover, we note that when the full $\varepsilon^{t, 111}-\sin ^{2} \psi$ curves are considered, we computed from Fig. $3 \mathrm{~b}$ the Young modulus and found 90.5 and 88.3 $\mathrm{GPa}$ for the as-grown and post-annealed samples, respectively; This matches very well the reference value of 89.7 GPa [51]. When the stress is determined with only $\varepsilon_{\perp}^{111}$, the error is estimated to lie between 10 and $20 \%$.

The tests were performed at $293 \mathrm{~K} \approx 0.22 T_{m}$ and the post-annealing corresponded to $473 K \approx 0.35 T_{m}$, where $T_{m}$ is the melting temperature of gold. However, the melting point of a crystal depends on the surface orientation, and this is accentuated in thin films, specifically with columnar grains: according to [55] the $\{111\}$ surface orientation (the more stable) lowered from $1337 K$ in bulk to $1167 \mathrm{~K}$ for $50 \mathrm{~nm}$-thick $\{111\}$ free-standing gold films. Finally, note that the estimated glass transition temperature of Kapton starts at approximately $630 \mathrm{~K}$, far enough from the present annealing.

The consequent decrease of the FWHM $w$, either by a mechanical $(\Delta w=10 \%$ for a true strain of $\epsilon=1.5 \%$ ) or by a thermal annealing $\left(\Delta w=30 \%\right.$ for 3 hours at $\left.200^{\circ} C\right)$, reveals that these nanocrystalline thin films were initially largely unstable from the thermodynamic point of view. Indeed, it is well-known that films grown by physical vapor deposition present an unusually high concentration of point defects [56, 57]. Moreover, the annealing temperature may be very critical for nanocrystalline materials, as is evidenced for electrodeposited nanocrystalline nickels in 
[58]: one hour at $150^{\circ} \mathrm{C}$ (resp. $200^{\circ} \mathrm{C}$ ) leads to negligible (resp. substantial) grain growth and an increase (resp. decrease) by $9.6 \%$ (resp. $28 \%$ ) of the yield strength. In [10], a slight strength increase was observed after an annealing at $100^{\circ} \mathrm{C}$ for one hour in nanocrystalline nickel; this was attributed to dislocation exhaustion. In our case, because the annealing suppressed the residual stress, the total stress at a given true strain is always more tensile for the post-annealed sample. We see here the importance to know the residual stress of the sample. Another effect reported in the literature was grain rotation: in [27] an annealing at $300^{\circ} \mathrm{C}$ for thirty minutes resulted in serious grain growth (from 29 to $57 \mathrm{~nm}$ ) and a large increase in twin boundaries (from 18 to $70 \%$ of $\Sigma 3$ boundaries). We will discuss it in the following paragraph.

Concerning the microstructure, the two gold thin films have thus equal thickness, similar grain size but different initial defect content and the as-grown sample has a compressive residual stress of about $200 M P a$. It is also possible that the thermal annealing induced a slight detwinning: when zooming in Fig. 1c close to $\psi \approx 39^{\circ}$, a small hump is seen in the as-grown diffracting volume and not in the post-annealed one. This corresponds to inclined twins, that is those with twin boundaries not parallel to the film surface. However, it is associated to a minute volume (less than $3 \%$ of the isotropic texture component at this angle), and so we consider it as negligible for the macroscopic response of the material. Moreover, within experimental precision, this hump remains constant during the test. The twins with twin boundaries parallel to the surface cannot be detected due to the fibre texture. Finally, according to [59], when looking at the $\{111\}$ reflection, extrinsic (resp. intrinsic) stacking faults induce a peak centre of mass to shift toward low (resp. high) angles, that is the asymmetry increases (resp. decreases). The figure $5 \mathrm{~b}$ then suggests that the deposition induces intrinsic stacking faults that the annealing deletes, whereas the mechanical test induces extrinsic stacking faults for both samples. However, a definitive statement is difficult because the small asymmetry detected here might have other causes.

Let us now compare their mechanical properties. First, their different work-hardening behaviours are clearly seen in Fig. 4a. We estimate the tangent modulus from the stress-strain curves presented in Fig. 3b (error bars result from different discrete tangent approximations on these curves). At an applied strain of $\epsilon=1.5 \%$, the tangent modulus is $13 \pm 2 \mathrm{GPa}$ for the as-grown sample, $1 \pm 2 \mathrm{GPa}$ for the post-annealed. This is a first indication that plasticity is either easily activated or at least not prevented in the post-annealed sample. Then, the relaxation of the two samples is also very different, see Fig. 4b-d. Strikingly, the relaxation of the post-annealed sample is almost two times smaller than the one of the as-grown sample for a true strain of $\epsilon=0.3 \%$, but both coincide at $\epsilon=1.5 \%$. In terms of total stress, the activation volume is about five times larger for the annealed sample at $\sigma_{\|}^{t}=200 \mathrm{MPa}$, but converges to $20-25 b^{3}$ for both samples at the end of the test. As discussed e.g. in [3], a high activation volume is associated with localized strain, typically the glide of a relatively long dislocation through the grain; on the contrary, a small activation volume corresponds to a set of numerous but small plastic events, like diffusion, grain boundary processes and/or glide of short dislocation segments. Finally, the evolution of the Bragg peak shape presents also large contrasts: whereas the FWHM of the as-grown sample decreases by $10 \%$ between $\epsilon=0$ and $\epsilon=1.5 \%$, the one of the post-annealed increases by $5 \%$ in the same interval. The FWHM of a Bragg peak depends on grain size and microstrain, but at $\psi=0^{\circ}$, the grain size cannot much increase because these grains are already columnar after deposition; thus, it is clear that the as-grown sample shows a stressdriven annealing, in which some defects are brought out of the grain, whereas the post-annealed sample presents a conventional plasticity behaviour, where dislocations are nucleated and generate localized elastic strain concentration.

It seems that all the differences observed here in the mechanical behaviours between the two samples may be explained with the initial point defect content, that lead to strong point defect-dislocation interactions in the asgrown sample but to easy glide of the dislocations after the thermal annealing. Indeed, in face-centered cubic crystals, dislocations glide on the $\{111\}$ planes, but in these $\{111\}$ fibre-textured films, the Schmid factor vanishes in the planes parallel to the specimen surfaces. Hence, dislocations glide in the other planes of the $\{111\}$ family, whose normal are inclined at $\arccos (1 / 3) \approx 70.5^{\circ}$ from the specimen surface normal. Moreover, in nanocrystalline metals, it is well-known that grain boundaries act as dislocation sources and sinks $[60,61,62,63]$. It is easily shown for these $50 \mathrm{~nm}$-thick films that as soon as the grain diameter is larger than $18 \mathrm{~nm}$, a dislocation emitted at the interface between the substrate and a grain boundary glides up to the free surface and not into the opposite grain boundary. For dislocations emitted in the thickness of the film (but still in a grain boundary), the probability that the glide plane encounters the surface is still higher. Given the grain diameter in the present thin films $(50-70 \mathrm{~nm})$, this shows that most probably dislocations, if not anchored in the grains, escape the grains and emerge at the free surface or at the interface with the substrate, or the other way around (from surfaces to grain boundaries). Note that the goldpolyimide interface is weak and does not obstruct the glide of dislocations [64]. This explains the difference in workhardening and activation parameters of the two samples: in the as-grown sample, the defect concentration is high so that dislocations are most often anchored inside the grains and the flow stress is governed by point defect-dislocation and dislocation-dislocation interactions, so that the activation volumes are small. On the contrary, the annealing has greatly lowered the defect density of the second sample, so that dislocations 'easily' cross the grains, and the flow stress is mainly dictated by the one necessary to nucleate 


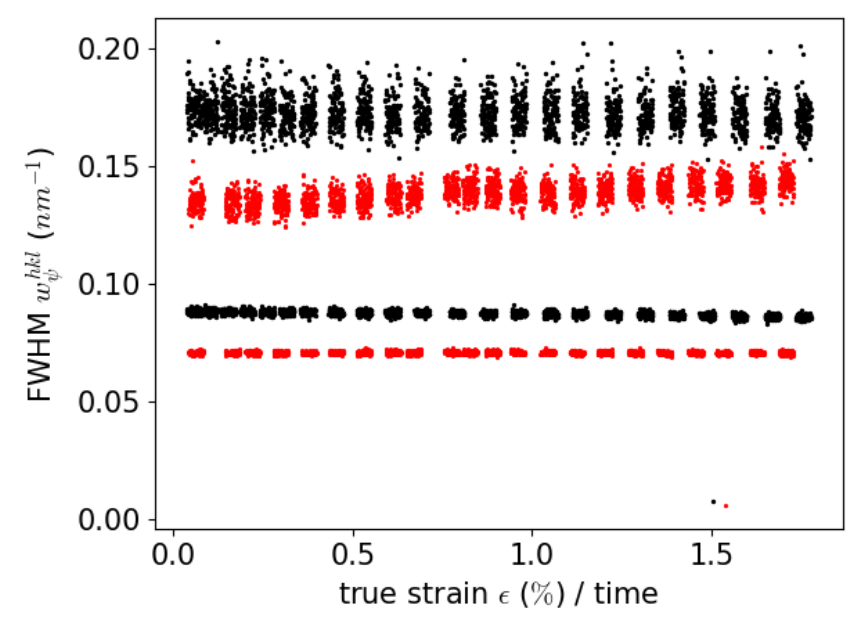

Figure 6: Bragg peak width for large tilt angles. Same abscissa as Fig. 3b. The black and red curves correspond to the as-grown and post-annealed samples, respectively.

new dislocations; the activation volumes remain high up to a much larger total stress (Fig. 4d), and decrease when dislocations get little by little trapped inside the grains.

One note of caution is necessary here, because it is known that when the grain size decreases a transition exists from perfect dislocation to single partial dislocation mediated plasticity. Chen et al proposes a model that quantifies the necessary shear stress to nucleate a perfect or a partial dislocation, taking the grain size as the source size [65]. In gold, this gives a transition length of approximately $40 \mathrm{~nm}$, which has been experimentally evidenced in single crystalline thin films, with the film thickness (projected on $\{111\}$ planes) as the source size [64]. Moreover, these authors observed with in-situ transmission electron microscopy that the thinner films $(40 \mathrm{~nm})$ had dislocations with small curvature radius and that were sometimes jogged, leading to ' $U$ ' or ' $S$ '-shape segments; cross-slips were also abundant and emissions of single partials were ascertained. All these plasticity behaviours were different in $160 \mathrm{~nm}$ thick films [64]. This raises questions about the Burgers vector we used in activation volume computations: it was the one of a perfect dislocation $(b \approx 0.288 \mathrm{~nm})$. The volume would be about 5 times larger had we chosen a Burgers vector corresponding to Shockley partials $\left(b_{p} \approx 0.166 \mathrm{~nm}\right)$. Moreover, the contribution of partial dislocations to the overall plastic strain probably depends on the strain, as the asymmetry of the peak may suggest. This leads to the conclusion that complementary experiments are needed to give a clear physical interpretation to activation volume values - see [66] for such a discussion in semiconductors, where the change of dislocation type associated to the brittle-to-ductile transition temperature is well established.

Another difficulty arises because a consistent activation rate theory is still missing when several plasticity mechanisms operate simultaneously. This is obviously the case here because (i) we are considering nanocrystals, (ii) the relaxation segments last for 10 minutes, (iii) we probe the micro- to macro-plasticity limit. Indeed, concerning (i), at this low strain rate, it is believed that dislocation nucleation controls the plastic strain rate [67]. But in nanocrystals, dislocation nucleation occurs at grain boundaries. Hence, grain boundary accommodation is necessary, and the consideration of a unique shear strain component, with a unique activation energy may be erroneous [68]. Second, (ii), the relaxation segments are long, inducing evolution in the microstructure, and thus possibly in the mechanisms. The apparent activation volumes we deduce are averages over theses microstructures. However, as evidenced in Fig 4a and 5a, neither the elastic (and so plastic) strain rate nor the FWHM changes their behaviours during the relaxation segments. Hence, change of dominating mechanisms after stress drops, as observed in [69] or more recently in [37] are excluded at this time scale (10 minutes) and resolution ( 5 seconds) in this study. Finally, (iii), another difficulty lies in the presence at the same time of grains lying in the elastic regime and ones in the plastic regime. Indeed, the plastic strain considered here is rather low. One can estimate the number of dislocations crossing each grain during the whole test. Let us first consider a grain in the main texture component. For an equibiaxial test, six slip systems have the same Schmid factor, $\sqrt{2} / 3 \sqrt{3} \approx 0.27$ (all other being zero). We suppose that the plastic strain in an in-plane direction is $1 \%$ and that this strain is equally distributed along the four slip systems that can contribute to this plastic strain. This implies e.g. $\varepsilon_{p}^{(\overline{11} 1)[101]}=0.5 \%$. For a grain diameter $d$ of $50 \mathrm{~nm}$, this leads to an elongation $\Delta L$ of $\varepsilon_{p}^{(\overline{11} 1)[101]} \times d=0.25 \mathrm{~nm}=0.87 \mathrm{~b}$. We obtain the same result in the other in-plane direction. As a conclusion, we can estimate that the nucleation of $8 \times 0.87 \approx 7$ perfect dislocations is necessary in each grain to induce an equibiaxial plastic strain of $1 \%$. For a more general grain orientation, the estimation proposed by [67] gives a number of 5 dislocations that crossed the grain (with a Taylor factor of 3). Notwithstanding the probably high dispersion in these estimations, this shows that a small proportion of grains is crossed by a dislocation during a strain jump or during the following relaxation segment, and the average over grains is thus difficult to interpret.

The FWHM shows that the as-grown sample cannot deforms only by conventional plasticity, i.e. nucleation and glide of dislocations. As proposed in [35], it is possible that dislocations are emitted and travel through the grain, bringing point defects out of the lattice (because the FWHM decreases), but, being progressively anchored, interact with each other (because the activation volumes decrease, in line with an increasing dislocation density). Note that the migration of point defects may also be triggered by the stress gradients, due either to the residual stress, or to the applied stress, considering that the grain boundaries are softer than grain interiors. However, these films are columnar, and with respect to [35] the thickness 
of the present film is much smaller, only $50 \mathrm{~nm}$, so that the other plasticity mechanisms mentioned in the introduction are favoured. The FWHM at high $\psi$ probes the micro-strain and the grain size in tilted directions. Figure 6 presents $w_{\psi=56^{\circ}}^{111}$ and $w_{\psi=65^{\circ}}^{220}$ (the other reflections were of no help: the $\{200\}$ reflection is very close to the $\{210\}$ peak of the $\mathrm{TiO} 2$, and the $\{311\}$ has too low intensity at large $\psi$ ). The decrease in peak width due to the thermal annealing is consistent with that observed in Fig. 5a, being $20 \%$ in both cases. The FWHM remains constant, so that elastic strain heterogeneities (either due to elastic anisotropy or to dislocations stored in the grains) are compensated possibly by a slight grain growth or by the ejection of point defects for the as-grown sample. Complementary transmission electron microscopy could help in clarifying this point. Note that the post-annealed sample is more prone to grain growth as its total stress is higher, and it is known that grain growth is governed by stress, and not strain $[34,70]$. Moreover, diffusion cannot be the leading plasticity mechanism, due to the value of the activation volume. It remains grain boundary sliding or grain boundary migration, and grain rotation. There is no reason here to exclude grain boundary motions because their signature would be difficult to note with X-ray diffraction. On the contrary, grain rotation seems to occur, as shown in Fig. 7, which presents the area of the Bragg peak, for the two samples and for several tilt angles. We observe that for the $\{111\}$ reflection at $\psi=0^{\circ}$, the area increases by $13 \%$ (resp. 5\%) for the as-grown sample (resp. for the post-annealed sample). The area of the peak is proportional to the diffracting volume, and it is clear in this figure that during the test the area decreases for moderate to high tilt angles but increases at the pole. Caution is necessary here because a small sample position realignment with the straining axis during the test could lead to artefacts [71]. However, as for the Bragg peak position, it was controlled with the calibrant's Bragg peaks, and their areas show no evolution. This indicates that grains, specifically in the as-grown sample, tend to align with their (111) planes parallel to the specimen surface. Grain rotation in gold thin films with microstructures very similar to the present ones were evidenced in [72], though at much larger strains.

\section{Conclusion}

Two sputtered deposited $\{111\}$ fibre-textured nanocrystalline gold $50 \mathrm{~nm}$-thin films have been compared at room temperature $\left(0.22 T_{m}\right)$, one being post-annealed at $0.35 T_{m}$. The most relevant points are:

- With synchrotron X-Ray diffraction, we observed that the Bragg peak width in the pole $\left(\psi=0^{\circ}\right)$ decreases either by a mechanical $(\Delta w=10 \%$ for a true strain of $\epsilon=1.5 \%)$ or by a thermal annealing ( $\Delta w=30 \%$ for 3 hours at $200^{\circ} \mathrm{C}$ ). On the contrary, far from the

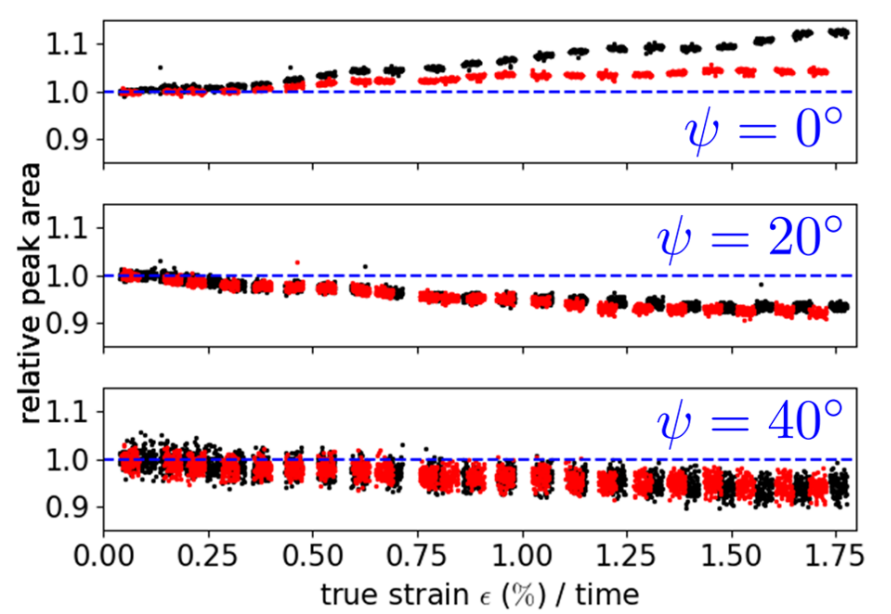

Figure 7: Normalized integrated intensity for the $\{111\}$ Bragg peak for the as-grown (black) and post-annealed (red) samples for different tilt $\psi$ angles, with the same abscissa as Fig. 3b. The horizontal blue lines are guides to the eye. Especially for the as-grown sample, the diffracting volume increases in the pole, at the expense of the diffracting volumes in the other directions.

pole, the FWHM dropped by $20 \%$ due to the thermal annealing, but the mechanical test has no effect. Given that the grains are columnar, this shows that these nanocrystalline thin films were largely unstable from the thermodynamic point of view, that moderate annealings took defects out of the grains, and that grain growth hardly ever occurs.

- For a given true strain, the out-of-plane applied elastic strain of the post-annealed sample was less negative than that of the as-grown sample (Fig. 4a); hence the in-plane plastic deformation of the former is larger, leading to the conclusion that the thermal annealing deleted defects that would have prevented the glide of dislocations.

- The as-grown sample presents work hardening, contrary to the post-annealed sample. This suggests dislocation-dislocation interactions in the first sample, but not in the second one.

- The Bragg peak area evolution suggests a slight grain rotation, stronger in the as-grown sample, but this must be confirmed.

- The work hardening behaviours are supported with the relaxation analysis (note that the logarithmic stress decay allows very good fits despite the duration of the relaxation segments that most probably leads to several mechanisms operating simultaneously, thus inducing small changes of the microstructure): The activation parameters are strongly different when compared at a given total stress (Fig. 4d, e.g. the activation volume is about five times larger for the annealed sample at $\sigma_{\|}^{t}=200 \mathrm{MPa}$ ); this agrees with a thermal 
annealing that much increases the mean free path of dislocations, and shows that diffusion controlled grain boundary activities cannot be the leading plasticity mechanisms.

- The decrease of the activation volume for the asgrown sample correlates well with an increasing obstacle density. It is nevertheless not fully understood why the activation volume decreases for the post-annealed sample. However only apparent activation volumes are characterized in this work, and the result may be different with physical activation volumes.

- We thus propose that dislocations mostly escape from the grains (they emerge at the free surface or at the substrate-film interface, or are stored in grain boundaries) in the post-annealed sample, but in the asgrown sample, dislocations are easily pinned at pointdefects, and this leads to dislocation-dislocation interactions.

\section{Acknowledgements}

The authors gratefully acknowledge SOLEIL for beam time allocation. This works was partially funded by the French Government program "Investissement d'Avenir" (LABEX INTERACTIFS, reference ANR-11-LABX-001701).

\section{References}

[1] Q. Wei, S. Cheng, K. Ramesh, E. Ma, Effect of nanocrystalline and ultrafine grain sizes on the strain rate sensitivity and activation volume: fcc versus bcc metals, Mater. Sci. Eng., A 381 (2009) 71-79.

[2] K. Jonnalagadda, N. Karanjgaokar, I. Chasiotis, J. Chee, D. Peroulis, Strain rate sensitivity of nanocrystalline Au films at room temperature, Acta Mater. 58 (2010) 4674-4684.

[3] S. Lee, S. J. Lee, B. C. D. Cooman, Work-hardening behavior of ultrafine-grained Mn transformation-induced plasticity steels, Acta Mater. 59 (2011) 7546-7533.

[4] G. Laplanche, J. Bonneville, C. Varvenne, W. A. Curtin, E. P. George, Thermal activation parameters of plastic flow reveal deformation mechanisms in the CrMnFeCoNi high-entropy alloy, Acta Mater. 143 (2018) 257-264.

[5] P. Feltham, Creep and stress relaxation in alpha-brass at low temperatures, Philos. Mag. 6 (62) (1961) 259-270.

[6] G. A. Sargent, Stress relaxation and thermal activation in niobium, Acta Met. 13 (1965) 663-671.

[7] J. L. Martin, B. Lo Piccolo, T. Kruml, J. Bonneville, Characterization of thermally activated dislocation mechanisms using transient tests, Mater. Sci. Eng. A 322 (2002) 118-125.

[8] D. Caillard, J. L. Martin, Thermally Activated Mechanisms in Crystal Plasticity, Pergamon, Amsterdam, 2003.

[9] Y. M. Wang, A. V. Hamza, E. Ma, Activation volume and density of mobile dislocations in plastically deforming nanocrystalline Ni, Appl. Phys. Lett. 86 (2005) 241917.

[10] Y. M. Wang, A. V. Hamza, E. Ma, Temperature-dependent strain rate sensitivity and activation volume of nanocrystalline Ni, Acta Mater. 54 (2006) 2715-2726.

[11] J. Hu, J. Zhang, Z. Jiang, X. Ding, Y. Zhang, S. Han, J. Sun, J. Lian, Plastic deformation behavior during unloading in compressive cyclic test of nanocrystalline copper, Mater. Sci. Eng. A 651 (2016) 999-1009.
[12] X. S. Yang, Y. J. Wang, G. Y. Wang, H. R. Zhai, L. H. Dai, T. Y. Zhang, Time, stress and temperature-dependent deformation in nanostructured copper: Stress relaxation tests and simulations, Acta. Mater. 108 (2016) 252-263.

[13] E. Hosseinian, M. Legros, O. N. Pierron, Quantifying and observing viscoplasticity at the nanoscale: Highly localized deformation mechanisms in ultrathin nanocrystalline gold films, Nanoscale 8 (2016) 9234-9244.

[14] M. Dao, L. Lu, R. L. Asaro, J. T. M. D. Hosson, E. Ma, Toward a quantitative understanding of mechanical behavior of nanocrystalline metals, Acta Mater. 55 (2007) 4041-4065.

[15] L. Mishnaevsky Jr., E. Levashov, Micromechanical modelling of nanocrystalline and ultrafine grained metals: A short overview, Comput. Mater. Sci. 96 (2015) 365-373.

[16] X. L. Wu, E. Ma, Dislocations in nanocrystalline grains, Appl. Phys. Lett. 88 (2006) 231911.

[17] J. H. Lee, T. B. Holland, A. K. Mukherjee, X. Zhang, H. Wang, Direct observation of Lomer-Cottrell Locks during strain hardening in nanocrystalline nickel by in situ TEM, Sci. Rep. 3 (2013) 1061.

[18] M. S. Colla, B. Amin-Ahmadi, H. Idrissi, L. Malet, S. Godet, J. P. Raskin, D. Schryvers, T. Pardoen, Dislocation-mediated relaxation in nanograined columnar palladium films revealed by on-chip time resolved HRTEM testing, Nat. Commun. 6 (2015) 5922 .

[19] R. A. Masumura, P. M. Hazzledine, C. S. Pande, Yield stress of fine grained materials, Acta Mater. 46 (1998) 4257-4534.

[20] Z. Shan, E. A. Stach, J. M. K. Wiezorek, J. A. Knapp, D. M. Follstaedt, S. X. Mao, Grain boundary-mediated plasticity in nanocrystalline Ni, Science 305 (2004) 654.

[21] X. Wei, J. W. Kysar, Residual plastic strain recovery driven by grain boundary diffusion in nanocrystalline thin films, Acta Mater. 59 (2011) 3937-3945.

[22] J. Schiotz, F. D. Tolla, K. Jacobsen, Softening of nanocrystalline metals at very small grain sizes, Nature 391 (1998) 561-563.

[23] D. S. Gianola, S. Van Petegem, M. Legros, S. Brandstetter, H. Van Swygenhoven, K. J. Hemker, Stress-assisted discontinuous grain growth and its effect on the deformation behavior of nanocrystalline aluminum thin films, Acta Mater. 54 (2006) $2253-2263$.

[24] N. Q. Vo, R. S. Averback, P. Bellon, S. Odunuga, A. Caro, Quantitative description of plastic deformation in nanocrystalline $\mathrm{Cu}$ : Dislocation glide versus grain boundary sliding, Phys. Rev. B 77 (2008) 134108.

[25] Y. B. Wang, B. Q. Li, M. L. Sui, S. X. Mao, Deformationinduced grain rotation and growth in nanocystalline $\mathrm{Ni}$, Appl. Phys. Lett. 92 (2008) 011903.

[26] A. Kobler, A. Kashiwar, H. Hahn, C. Kubel, Combination of in situ straining and ACOM TEM: A novel method for analysis of plastic deformation of nanocrystalline metals, Ultramicroscopy 128 (2013) 68-81.

[27] P. F. Rottmann, K. J. Hemker, Experimental quantification of mechanically induced boundary migration in nanocrystalline copper films, Acta Mater. 140 (2017) 46-55.

[28] F. H. Dalla Torre, E. V. Pereloma, C. H. J. Davies, Strain rate sensitivity and apparent activation volume measurements on equal channel angular extruded $\mathrm{Cu}$ processed by one to twelve passes, Scripta Mater. 51 (2004) 367-371.

[29] S. Van Petegem, S. Brandstetter, H. Van Swygenhoven, Internal and effective stresses in nanocrystalline electrodeposited $\mathrm{Ni}$, Appl. Phys. Lett. 89 (2006) 073102.

[30] L. Lu, T. Zhu, Y. Shen, M. Dao, K. Lu, S. Suresh, Stress relaxation and the structure size-dependence of plastic deformation in nanotwinned copper, Acta. Mater. 57 (2009) 5165-5173.

[31] G. Mohanty, J. Wehrs, B. L. Boyce, A. Taylor, M. Hasegawa, L. Philippe, J. Michler, Room temperature stress relaxation in nanocrystalline $\mathrm{Ni}$ measured by micropillar compression and miniature tension, J. Mater. Res. 31 (2016) 1085-1095.

[32] G. Lemoine, L. Delannay, H. Idrissi, M. S. Colla, T. Pardoen, Dislocation and back stress dominated viscoplasticity in freestanding sub-micron Pd films, Acta. Mater. 111 (2016) 10-21. 
[33] J. Wehrs, M. J. Deckarm, J. M. Wheeler, X. Maeder, R. Birringer, S. Mischler, J. Michler, Elevated temperature, micro-compression transient plasticity tests on nanocrystalline Palladium-Gold: Probing activation parameters at the lower limit of crystallinity, Acta. Mater. 117 (2017) 124-137.

[34] K. Zhang, J. Weertman, J. A. Eastman, Rapid stress-driven grain coarsening in nanocrystalline $\mathrm{Cu}$ at ambient and cryogenic temperatures, Appl. Phys. Lett. 87 (2005) 061921.

[35] P. Godard, P. O. Renault, D. Faurie, D. Thiaudière, Relaxation mechanisms in a gold thin film on a compliant substrate as revealed by X-ray diffraction, Appl. Phys. Lett. 110 (2017) 211901.

[36] Z. Budrovic, H. Van Swygenhoven, P. M. Derlet, S. Van Petegem, B. Schmitt, Plastic deformation with reversible peak broadening in nanocrystalline nickel, Science 304 (2004) 273.

[37] Z. Sun, S. Van Petegem, A. Cervellino, K. Durst, W. Blum, $\mathrm{H}$. Van Swygenhoven, Dynamic recovery in nanocrystalline $\mathrm{Ni}$, Acta Mater. 91 (2015) 91-100.

[38] J. Lohmiller, R. Baumbusch, O. Kraft, P. Gruber, Differentiation of deformation modes in nanocrystalline Pd films inferred from peak asymmetry evolution using In Situ x-ray diffraction, Phys. Rev. Lett. 110 (2013) 066101.

[39] Y. M. Wang, R. T. Ott, A. V. Hamza, M. F. Besser, J. Almer, M. J. Kramer, Achieving large uniform tensile ductility in nanocrystalline metals, Phys. Rev. Lett. 105 (2010) 215502.

[40] B. Chen, K. Lutker, J. Lei, J. Yan, S. Yang, H. K. Mao, Detecting grain rotation at the nanoscale, Proc. Natl. Acad. Sci. USA 111 (2014) 3350-3353.

[41] K. Mongkolsuttirat, J. Smyth, W. Brown, R. Vinci, The effect of grain size on viscoelastic relaxation behavior of Au thin films, Scripta Mater. 155 (2018) 1-4.

[42] S. Djaziri, D. Faurie, P. O. Renault, E. Le Bourhis, P. Goudeau, G. Geandier, D. Thiaudière, Yield surface of polycrystalline thin films as revealed by non-equibiaxial loadings at small deformation, Acta Mater. 61 (2013) 5067-5077.

[43] D. Collins, M. Mostafavi, R. Todd, T. Connolley, A. Wilkinson, A synchrotron X-ray diffraction study of in situ biaxial deformation, Acta Mater. 90 (2015) 46-58.

[44] M. V. Upadhyay, S. Van Petegem, T. Panzner, R. A. Lebensohn, H. Van Swygenhoven, Study of lattice strain evolution during biaxial deformations of stainless steel using a finite element and fast Fourier transform based multiscale approach, Acta Mater. 118 (2016) 28-43.

[45] G. Geandier, D. Thiaudière, R. N. Randriamazaoro, R. Chiron, S. Djaziri, B. Lamongie, Y. Diot, E. Le Bourhis, P. O. Renault, P. Goudeau, A. Bouaffad, O. Castelnau, D. Faurie, F. Hild, Development of a synchrotron biaxial tensile device for in situ characterization of thin films mechanical response, Rev Sci Instrum 81 (2010) 103903

[46] G. Besnard, F. Hild, S. Roux, "Finite-element" displacement fields analysis from digital images: Application to Portevin-Le Châtelier bands, Exp Mech 46 (2006) 789-803.

[47] S. Djaziri, P. O. Renault, F. Hild, E. Le Bourhis, P. Goudeau, D. Thiaudière, D. Faurie, Combined synchrotron x-ray and image correlation analyses of biaxially deformed $\mathrm{W} / \mathrm{Cu}$ nanocomposite thin films on Kapton, J. Appl. Crystallogr. 44 (2011) 1071-1079.

[48] P. O. Renault, T. Sadat, P. Godard, W. He, P. Guerin, G. Geandier, N. Blanc, N. Boudet, P. Goudeau, Continuous cyclic deformations of a $\mathrm{Ni} / \mathrm{W}$ film studied by synchrotron X-ray diffraction, Surf. Coat. Technol. 332 (2017) 351-357.

[49] G. Geandier, D. Faurie, P. O. Renault, D. Thiaudière, E. Le Bourhis, In situ monitoring of X-ray strain pole figures of a biaxially deformed ultra-thin film on a flexible substrate, J. Appl. Cryst. 47 (2014) 181-187.

[50] U. Welzel, A. Kumar, E. Mittemeijer, Extremely anisotropic, direction-dependent elastic grain interaction: The case of ultrathin films, Appl. Phys. Lett. 95 (2009) 111907.

[51] D. Faurie, P. O. Renault, E. Le Bourhis, P. Goudeau, Study of texture effect on elastic properties of au thin films by x-ray diffraction and in situ tensile testing, Acta Mater. 54 (2006)
4503-4513.

[52] Q. Wei, Strain rate effects in the ultrafine grain and nanocrystalline regimes - influence on some constitutive responses, J. Mater. Sci. 42 (2007) 1709-1727.

[53] C. Duhamel, Y. Bréchet, Y. Champion, Activation volume and deviation from Cottrell-Stokes law at small grain size, Int. J. Plast 26 (2010) 747-757.

[54] T. Ida, New measures of sharpness for symmetric powder diffraction peak profiles, J. Appl. Cryst. 41 (2008) 393-401.

[55] R. Sankarasubramanian, K. Kumar, Effects of surface anisotropy on the melting temperatures of free-standing gold nanofilms, Comput. Mater. Sci. 49 (2010) 386-391.

[56] F. M. D'Heurle, J. M. E. Harper, Note on the origin of intrinsic stresses in films deposited via evaporation and sputtering, Thin Solid Films 171 (1989) 81-92.

[57] E. Chason, P. R. Guduru, Tutorials: Understanding residual stress in polycrystalline thin films through real-time measurements and physical models, J. Appl. Phys. 19 (2016) 191101.

58] Y. M. Wang, S. Cheng, Q. M. Wei, E. Ma, T. Nieh, A. Hamza, Effects of annealing and impurities on tensile properties of electrodeposited nanocrystalline Ni, Scipta Mater. 51 (2004) 10231028.

[59] A. I. Ustinov, L. O. Olikhovska, N. M. Budarina, F. Bernard, Line profile fitting; the case of fcc crystals containing stacking faults, in: Diffraction Analysis of the Microstructure of Materials, Springer, 2004, Ch. 13.

[60] R. Z. Valiev, V. Y. Gertsman, O. A. Kaibyshev, Grain boundary structure and properties under external influences, Phys. Stat. Sol (a) 97 (1986) 11-56.

[61] V. Yamakov, D. Wolf, M. Salazar, S. R. Phillpot, H. Gleiter, Length-scale effects in the nucleation of extended dislocations in nanocrystalline $\mathrm{Al}$ by molecular-dynamics simulation, Acta Mater. 49 (2001) 2713-2722.

[62] H. Van Swygenhoven, P. M. Derlet, A. G. Frøseth, Nucleation and propagation of dislocations in nanocrystalline fcc metals, Acta Mater. 54 (2006) 1975-1983.

[63] F. Mompiou, M. Legros, A. Boé, M. Coulombier, J. P. Raskin, T. Pardoen, Inter- and intragranular plasticity mechanisms in ultrafine-grained Al thin films: An in situ TEM study, Acta Mater. 61 (2013) 205-216.

[64] S. Oh, M. Legros, D. Kiener, P. Gruber, G. Dehm, In situ TEM straining of single crystal Au films on polyimide: Change of deformation mechanisms at the nanoscale, Acta Mater. 55 (2007) $5558-5571$.

[65] M. Chen, E. Ma, K. Hemker, H. Sheng, Y. Wang, X. Cheng, Deformation twinning in nanocrystalline aluminium, Science 300 (2003) 1275-1277.

[66] J. M. Wheeler, L. Thilly, A. Morel, A. A. Taylor, A. Montagne, R. Ghisleni, J. Michler, The plasticity of indium antimonide: Insights from variable temperature, strain rate jump micro-compression testing, Acta Mater. 106 (2016) 283-289.

[67] G. Saada, T. Kruml, Deformation mechanisms of nanograined metallic polycrystals, Acta. Mater. 59 (2011) 2565-2574.

[68] U. F. Kocks, A. S. Argon, M. F. Ashby, Thermodynamics and kinetics of slip, Prog. Mater. Sci. 19 (1975) 1.

[69] W. Blum, A. Rosen, A. Cegielska, J. L. Martin, Two mechanisms of dilocation motion during creep, Acta. Metall. 37 (1989) $2439-2453$.

[70] T. Rupert, D. Gianola, Y. Gan, K. J. Hemker, Experimental observations of stress-driven grain boundary migration, Science 326 (2009) 1686-1690.

[71] F. Mompiou, M. Legros, Quantitative grain growth and rotation probed by in-situ TEM straining and orientation mapping in small grained Al thin films, Scripta Mater. 99 (2015) 5-8.

[72] P. Liu, S. C. Mao, L. H. Wang, X. D. Han, Z. Zhang, Direct dynamic atomic mechanisms of strain-induced grain rotation in nanocrystalline, textured, columnar-structured thin gold films, Scripta Mater. 64 (2011) 343-346.

[73] A. H. Cottrell, R. J. Stokes, Effects of temperature on the plastic properties of aluminium crystals, Proc. R. Soc. Lond. A 233 (1955) 17-34. 


\section{APPENDIX}

We derive here the logarithmic decay of the shear stress during a relaxation test, and the prefactor that leads to the apparent activation volume. The following derivation is largely inspired by [6] but adapted to a case where the strain is not imposed directly by a tensile machine but by the substrate, eliminating the elastic interaction with the machine. Moreover, we made an effort to state as clearly as possible all the assumptions used.

The displacement of the thin film is supposed constant after a load jump. Its length $l$ thus satisfies

$$
d l=0 .
$$

The evolution of the length is thus equilibrated between elastic and plastic elongations, $d l_{e}$ and $d l_{p}$, respectively:

$$
d l_{e}+d l_{p}=0 .
$$

We thus have for any time $t$

$$
\epsilon_{p}(t):=\frac{d l_{p}(t)}{l}=-\frac{d l_{e}(t)}{l}=-C \sigma(t)
$$

where $\sigma$ is the total stress and $C$ is a test-dependent combination of the elastic constants. In a polycrystal, the uniaxial stress (resp. strain) $\sigma$ (resp. $\epsilon$ ) is related to the shear stress (resp. strain) $\tau$ (resp. $\gamma$ ) with a Taylor factor $M$ :

$$
\begin{aligned}
& \sigma=M \tau \\
& \gamma=M \epsilon .
\end{aligned}
$$

Because uniaxial and equibiaxial tests lead to the same von Mises stresses, we suppose that these relations apply for the tests presented in the manuscript. Hence Eq. A-3 can be translated into a shear stress-shear strain relation:

$$
\gamma_{p}(t)=-C M^{2} \tau(t)
$$

The first hypothesis is that the shear strain is thermally activated, so there exists an activation energy $G$ such that

$$
\dot{\gamma}_{p}=\nu \exp \left(-\frac{G}{k T}\right)
$$

where $\nu$ depends on the 'efficiency' of the plasticity mechanisms, the volume density of sites at which thermal activation occurs and the atomic frequency. This form of thermal activation neglects reverse jumps, and this should be reasonable at the temperature and stress levels considered here [68]. Combining Eq. A-6 and A-7 gives

$$
\dot{\tau}(t)=\frac{-\nu}{C M^{2}} \exp \left(-\frac{G}{k T}\right) .
$$

The second hypothesis assumes that the activation energy during the relaxation regime is an affine function of the effective stress:

$$
G\left(T, \tau^{*}\right)=G^{*}(T)-V^{*}(T) \tau^{*}(T) .
$$

This is nothing more than a first order Taylor expansion, valid for small enough stresses.

The third hypothesis is the law of Cottrell and Stokes [73], who noticed experimentally that the effective stress and the total stress are proportional:

$$
\tau^{*}=\frac{\tau}{\alpha} .
$$

The last three equations lead to

$$
\frac{d \tau^{*}(t)}{d t}=B \exp \left(C \tau^{*}\right)
$$

with

$$
B=\frac{-\nu}{C M^{2} \alpha} \exp \left(-\frac{G^{*}}{k T}\right)
$$

and

$$
C=\frac{V^{*}}{k T}
$$

The solution of this equation with the initial condition $\tau^{*}(0)=\tau_{0}^{*}$ is

$$
\tau^{*}(t)=\tau_{0}^{*}-\frac{1}{C} \ln \left[-C B \exp \left(C \tau_{0}^{*}\right) t+1\right]
$$

or

$\tau^{*}(t)-\tau_{0}^{*}=-\frac{k T}{V^{*}} \ln \left[\frac{V^{*} \nu}{k T C M^{2} \alpha} \exp \left(\frac{-G^{*}+V^{*} \tau_{0}^{*}}{k T}\right) t+1\right]$

showing that the prefactor is inversely proportional to the activation volume.

Our derivation supposes that the microstructure is constant all over the relaxation segment. Rigorously, this should not be the case (e.g. due to Orowan equation and the continuity of the dislocation speed), and this is why the activation volume $V^{*}$ considered in this appendix is usually termed the apparent activation volume. To reach the physical activation volume, defined by Eq. A-9 for constant internal stress, the methods developed in $[7,8]$ are necessary. 